\title{
Robust Hybrid Control Algorithm for Tuning the Altitude and Attitude of Unmanned Aerial Vehicle
}

\author{
Bohang Wang (iD) and Daobo Wang \\ College of Automation Engineering, Nanjing University of Aeronautics and Astronautics, Nanjing, China \\ Correspondence should be addressed to Bohang Wang; bhwang@nuaa.edu.cn
}

Received 27 August 2019; Accepted 23 October 2019; Published 25 January 2020

Academic Editor: L. Fortuna

Copyright ( $) 2020$ Bohang Wang and Daobo Wang. This is an open access article distributed under the Creative Commons Attribution License, which permits unrestricted use, distribution, and reproduction in any medium, provided the original work is properly cited.

\begin{abstract}
In this article, a new and novel robust hybrid control algorithm is designed for tuning the parameters of unmanned aerial vehicle (UAV). The quadrotor type UAV mathematical model is taken to observe the effectiveness of our designed robust hybrid control algorithm. The robust hybrid control algorithm consists of $H_{\infty}$ based regulation, pole-placement and tracking (RST) controller along with mixed sensitivity function is applied to control the complete model of UAV. The selected rotor craft is under-actuated, nonlinear and multivariable behavior in nature along with six degrees of freedom (DOF). Due to all these aforementioned issues its stabilization is quite difficult as compared to fully actuated systems. For the tuning of nonlinear parameters of the UAV, we designed, robust hybrid control algorithm is used. Moreover, the performance of the designed controller is compared with robust controller. The validity and effectiveness of the designed controllers are simulated in MATLAB and Simulink, in which the designed controller shows better steady state behavior, robustness and converges quickly in specific amount of time as compared to robust controller.
\end{abstract}

\section{Introduction}

Over the recent years, the demand of unmanned aerial vehicle (UAV) is increased rapidly due to playing a vital role in various fields, i.e., transportation, surveillance, firefighting, rescue and health and safety monitoring purposes [1]. Essentially, it is a robot without a humanoid pilot or crew on board that's why it is also called un-crewed or unmanned aerial vehicle. All the UAV's are equipped with a ground-based control station and the communication system to connect with each other [2]. This technology operates in two ways; under remote control by the machinist or autonomously by the onboard controller, but most of them are remotely operated. UAV's are commonly classified into two types first is fixed wing UAV's and second is multirotor based UAV's. Generally, fixed wing based UAV's are used for military and intelligence purposes [3].

The rotorcraft UAV's has a modest mechanical structure, its simple construction helps to perform a multiple task, for example, it can perform vertical take-off and landing (VTOL) as compared to fixed wing UAV's [4]. It can hover itself, remain in the air at one place, can do a stable flight and can certainly reach the places where humans are not easily accessible to reach. Now these days, this technology is widely used for the aerial photography, delivering the stuff, life support, protecting national borders, building inspection, monitoring of the environment and in other various fields [5]. Due to these qualities, a high attention has been paid for the research work on UAV's. Rotor crafts are highly nonlinear and multivariable in nature, having multiple input and multiple output (MIMO) system. But, due to some characteristics the dissimilar forms of uncertainties occur and is difficult to achieve an accurate system model because of the complexity. However, it can change its position and direction rapidly due to its maneuvering quality [6].

Rotorcrafts are classified into many categories i.e., dual propeller based rotorcraft called Bi-rotor, Tri-rotor, Quadrotor, Hex-rotor, and Octa-rotorcrafts [7]. In this research our main focus is on quadrotor aerial vehicle, it is an under-actuated type of rotorcraft. Because it has four actuators and overall system has six degrees of freedom (DOF) [8], three for the Earths fixed frame and three in the fixed body of UAV. So if the number of actuators is less than the DOF the system is said to be an under-actuated system. It has four control inputs just same like that of the classical helicopter model, having altitude control, 
longitudinal control, lateral control and angular moment control. The outputs of quadrotor rotorcraft contain linear velocities, angular velocities, Euler angles and its positions [9]. Moreover, the linear and angular subsystems are integrated to make a successive control system. For the best flight performance of UAV, it needs a better structure along with the better controller, which might be able to make the complete scenario with greater stability. Formerly, different control schemes were designed which are able to control the whole dynamics of UAV as well they showed better robustness. In which proportional integral derivative (PID) controller, model reference adaptive control (MRAC), sliding mode controller (SMC) and different robust control schemes were used [10-12].

Moreover, the uncertainty issues could be easily handled by, PID control, MRAC, back stepping, model predictive control (MPC) etc. The proportional derivative (PD) controller in [13], it cannot resolve the errors produced due to the rapid change in parameters of UAV. In [14], fuzzy control logic combined with PD, PID controller to control the altitude and attitude of UAV and solve these errors due to the rapid change. Previously, chattering problem was efficiently reduced by combining SMC with fuzzy control system. Back stepping technique was used to decay the complex nonlinear system. A back-stepping control scheme was applied to control the uncertainty issues and can also solve the computational expansion problems easily due to base on the first-order instruction filtering. The robust regulation, pole-placement, tracking (RST) controller, the robust $H_{\infty}$ controller was designed by Landau [15], and Fortuna [16]. Previously different RST control algorithms were designed to control the 6-DOF complex behavior of UAV [17-21].

Many of the aforementioned problems were previously discussed and solved by using different control schemes. However, the disturbances are caused during the flight to decrease the system disturbances, uncertainties, in this paper, we design the dual control strategy to resolve the aforementioned problems, by designing the "Robust $H_{\infty}$ controller". The RST controller is combined with $H_{\infty}$ controller for better tuning of the altitude and attitude of UAV. However, the robust RST controller is responsible for the fine tuning of the system and it works on the basis of sensitivity. The control structure is used to enhance the infinite norms of some constraints in transfer function (TF) approach. It is used to lessen the parameter perturbation and modeling error which have an effect on the control performance and improves the robustness of the system. Earlier, many researchers studied this control structure and then applied in the UAV's to examine and stabilize the attitude angle. According to the system requirements, many other systems are constructed for solving the problem.

The fundamental achievements in this research are as follows, (1) an innovative control strategy is designed to control the highly multivariable system of UAV; (2) $H_{\infty}$ controller with fine-tuning by the gains of the a robust RST controller; (3) robust RST controller works on the basis of the sensitivity function in the whole system (4) steady state characteristics of the proposed model of the UAV is seen realistically from the experimental perspective.

This research article divided into six different sections. Section 1 defines the introduction, Section 2 discussed the mathematical modeling of quadrotor, which is followed by

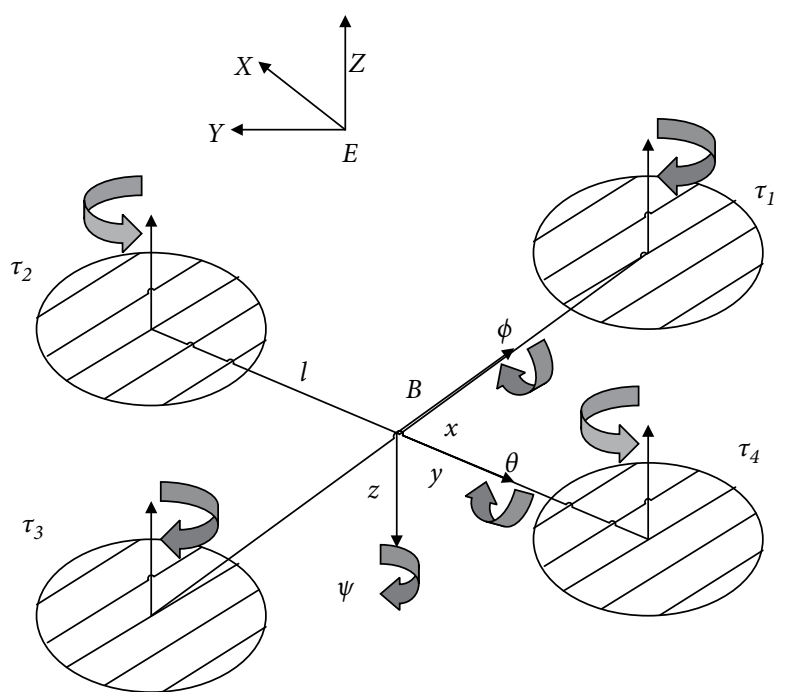

FIGURE 1: Model of quadrotor UAV [22].

Section 3, in which designing of controller is demonstrated. In Section 4, the modeling of control objectives is discussed. However, robust hybrid controller along with mixed sensitivity is defined in Section 5. Section 6 presents the simulation result and discussion. Lastly, the whole manuscript is concluded in Section 7.

\section{Modeling of Quadrotor UAV}

In this section the complete mathematical model of quadrotor aerial vehicle is discussed, whereas the equation of motions of quadrotor UAV is considered by using Newton's law of motion. It defines that the addition of all external forces that are acting on the body of UAV time period oscillations of the body momentum [14]. However, the addition of all forces in the body of quadrotor is equal to the change of rate of its rotational movements. The relationship between the Earth's co-ordinate system and fixed body frame can be defined in terms of rotational matrix i.e.

$$
R(\varphi, \theta, \psi)=\left[\begin{array}{ccc}
c \theta c \psi & c \psi s \theta s \varphi-s \psi c \theta & c \psi s \theta c \varphi+s \psi s \varphi \\
c \theta s \psi & s \psi s \theta s \varphi+c \psi c \varphi & s \psi s \theta c \varphi-c \psi s \varphi \\
-s \theta & c \theta s \varphi & c \theta c \varphi
\end{array}\right]
$$

whereas, $c=\cos ; s=\sin$

Figure 1, defines the model of quadrotor UAV. Now, the devolved thrust by rotors of UAV can be expressed as;

$$
\tau_{n}=C_{t_{n}} \delta_{n},
$$

whereas, $n=\{1, . ., 4\}$ " $\tau_{n}$ " is the produced thrust, " $C_{t_{n}}$ " is the coefficient of thruster and " $\delta_{n}$ " is the rotational velocity that is produced by the rotors of UAV. In the fixed body frame, the upward direction or lift is produced by each rotor in $z$-direction the altitude toward the body fixed frame. The resultant forces are as follows;

$$
f_{B}=\left[\begin{array}{lll}
0 & 0 & \sum_{n=1}^{4} \tau_{n}
\end{array}\right]^{T} .
$$


By transforming these forces in Earth's co-ordinate system that can be written as;

$$
\left\{\begin{array}{c}
f_{E}=R(\varphi, \theta, \psi) * f_{B} \\
f_{E}=\left[\begin{array}{l}
\sum_{n=1}^{4} \tau_{n}(c \psi s \theta c \varphi+s \psi s \varphi) \\
\sum_{n=1}^{4} \tau_{n}(s \psi s \theta c \varphi-c \psi s \varphi) \\
\sum_{n=1}^{4} \tau_{n}(c \theta c \varphi)
\end{array}\right]
\end{array}\right.
$$

Moreover, the mass or weight of the UAV is subjected directly to the gravity " $m_{g}$ " downwards along with the Earth's co-ordinate system.

Assumption I. By considering the wind resistance during the flight, the relationship between the rotorcraft and the wind speed in UAV fixed body frame system is established as follows;

$$
W_{E}=\left[\begin{array}{lll}
W_{x} & W_{y} & W_{z}
\end{array}\right]^{T}=\left[\begin{array}{ccc}
-\frac{1}{2} & \left|V_{x}\right| V_{x} & C_{w x} \\
-\frac{1}{2} & \left|V_{y}\right| V_{y} & C_{w y} \\
-\frac{1}{2} & \left|V_{z}\right| V_{z} & C_{w z}
\end{array}\right]
$$

In Equation (5), $\left(V_{x}, V_{y}, V_{z}\right)$ are the velocities, and $\left(C_{w x}, C_{w y}, C_{w z}\right)$ are the drag coefficient along $X, Y, Z$ direction.

Remark I: The length of each arm of UAV is " $l$ " and the roll moments " $M_{\varphi}$ " is generated by the difference of each rotor rotation per minute (RPM) or speed which can be stated as;

$$
\left[\begin{array}{c}
M_{\varphi} \\
M_{\theta} \\
M_{\psi}
\end{array}\right]=\left[\begin{array}{c}
l\left(\tau_{2}-\tau_{4}\right) \\
l\left(\tau_{1}-\tau_{3}\right) \\
l * d\left(\delta_{2}^{1}+\delta_{3}^{2}-\delta_{2}^{2}-\delta_{4}^{2}\right)
\end{array}\right]
$$

whereas, " $d$ " is the coefficient of drag factor. Now, the altitude of UAV changes during the flight, the direction of rotors are changes respectively. At that time the each rotor will produce a falling moment due to gravity " $m_{g}$ ". It can be stated as;

$$
m_{g_{n}}=I_{r} \delta_{n} \text {. }
$$

In the above formula, " $I_{r}$ " is the inertial matrix of the rotor.

Assumption II: By assuming that UAV motion does not change the direction on the rotors along its rotational axis, it is in hovering mode. It means that its altitude and position are not changed.

Now, the rotor axes are aligned rigidly to the body of UAV, the rotational moment produced by the axis of rotor is equal to the rotational velocity of fixed body frame that can be stated as:

$$
\delta=[p, q, 0]^{T} .
$$

Assumption III: Let the inertia matrix " $I$ " of each rotor be written as;

$$
I_{r}=\left[\begin{array}{ccc}
I_{x} & 0 & 0 \\
0 & I_{y} & 0 \\
0 & 0 & I_{z}
\end{array}\right]
$$

Remark II: The moment produced by the gyro is " $m_{g_{n}}$ " and its total torque " $m_{g}$ " of a single rotor can be expressed as; $m_{g_{n}}=\left[\begin{array}{lll}-I_{z} q & I_{z} p & 0\end{array}\right]^{T} \delta_{n}$ and $m_{g}=\left[\begin{array}{lll}-I_{z} q & I_{z} p & 0\end{array}\right]^{T}$ $\left(\delta_{1}+\delta_{3}-\delta_{2}-\delta_{4}\right)$.

Now the gyroscopic inertial moments are;

$$
m_{g_{B}}=\left[\begin{array}{c}
\left(I_{y}-I_{z}\right) q r \\
\left(I_{z}-I_{x}\right) p r \\
\left(I_{x}-I_{y}\right) p q
\end{array}\right] .
$$

The quadrotor vehicle has 6-DOF, it is not easy to stabilize the dynamic behavior of quadrotor. The rotational velocity rates of the system are $(p, q, r)$ and the translational velocities are denoted by $(u, v, w)$ and its position is defined by $(x, y, z)$. However, the Euler angles of the vehicle are defined by $(\varphi, \theta, \psi)[23]$.

Assumption IV: By considering the relationship between the rotational velocity rates and attitude angles under small disturbances. To establish equation of motion of quadrotor aerial vehicle according to Earth's co-ordinate system is as follows:

$$
\left\{\begin{array}{l}
\left\{\begin{array}{l}
m \dot{x}=(c \psi s \theta c \varphi+s \psi s \varphi) u_{1} \\
m \dot{y}=(s \psi s \theta c \varphi-c \psi s \varphi) u_{1} \\
m \dot{z}=(c \theta c \varphi) u_{1}-m_{g}
\end{array}\right. \\
\left\{\begin{array}{l}
I_{x} \dot{\varphi}=\theta \psi\left(I_{y}-I_{z}\right) I_{z} \theta \delta+u_{2} \\
I_{y} \dot{\theta}=\varphi \psi\left(I_{z}-I_{x}\right)-I_{z} \varphi \delta+u_{3} \\
I_{z} \dot{\psi}=\varphi \theta\left(I_{x}-I_{y}\right)+u_{4}
\end{array}\right. \\
\left\{\begin{array}{l}
u_{1}=\sum_{n=1}^{4} \tau_{n} \\
u_{2}=l\left(\tau_{3}-\tau_{1}\right) \\
u_{3}=l\left(\tau_{4}-\tau_{2}\right) \\
u_{4}=d\left(\delta_{2}^{1}+\delta_{3}^{2}-\delta_{2}^{2}-\delta_{4}^{2}\right) \\
\delta=\delta_{1}+\delta_{3}-\delta_{2}-\delta_{4}
\end{array}\right.
\end{array}\right.
$$

\section{Designing of Dual Controller}

The complete control structure is defined in Figure 2. The overall two working loops of the control system one is for the desired position the external loop and second is for the desired speed is the inner loop that is shown in blocks. However, in Figure 3, it shows the inner working of control structure.

\section{1. $H_{\infty}$ Based Robust RST Controller with Mixed} Sensitivity. Figure--3, shows the block diagram of inner loop which could be control of the attitude of RST control based mixed sensitivity which is written as;

$$
\left\{\begin{array}{l}
R=\frac{P}{1-K P} \\
S=\frac{P}{1-K P} \\
T=\frac{K P}{1-K P}
\end{array}\right.
$$

where " $P$ " is the controlled position, " $K$ " is variable for the " $H_{\infty}$ " controller, " $d(t)$ " is the external disturbance added in the model of UAV and " $y(t)$ " is the output of the system, $d(t)=[0.15 \operatorname{Sin}(0.15 \pi t), 0.15 \operatorname{Cos}(0.15 \pi t), 0.15 \operatorname{Sin}(0.15 \pi t)]$. 


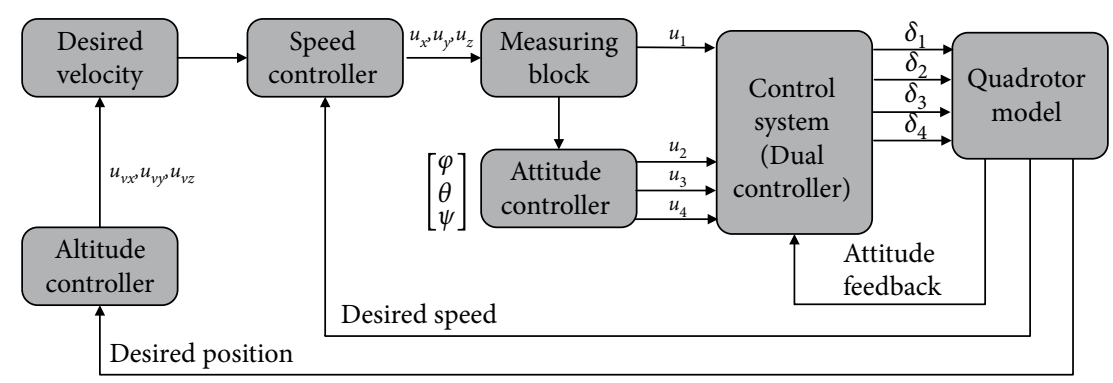

FIGURE 2: Block diagram of quadrotor UAV.

Assumption V: By considering the suitable weight functions of RST controller " $\left(w_{1}, w_{2}, w_{3}\right)$ " are mixed sensitivity functions which will satisfy the formula of " $H_{\infty}$ " and obtain controller " $K$ "as shown in Equation (12).

$$
\left\|\begin{array}{l}
w_{1} R \\
w_{2} S \\
w_{3} T
\end{array}\right\|_{\infty} \leq \beta
$$

In Equation (13), “ $\beta$ ” is the performance index and its value are approximately equal to unity. Now, the hybrid mathematical model of the RST controller with mixed sensitivity along with the weighing function can be expressed as;

$$
\begin{gathered}
{\left[\begin{array}{c}
z_{1} \\
z_{2} \\
e
\end{array}\right]=\left[\begin{array}{cc}
W_{1} & W_{1} P \\
0 & W_{2} P \\
I_{r} & W_{3} P
\end{array}\right]\left[\begin{array}{ll}
r & u
\end{array}\right]=G\left[\begin{array}{ll}
r & u
\end{array}\right]^{T} .} \\
u=K e .
\end{gathered}
$$

In formula (15), $z_{1}, z_{2}$ are weighing evaluation outputs, " $u$ " is the input of entire system, " $r$ " is the reference signal, " $K$ " is the $H_{\infty}$ controller and " $e$ " is the tracking error of system. Now the generalized control " $G$ " object and the controlled objects " $P(S)$ " are written as;

$$
\left\{\begin{aligned}
G & =\left[\begin{array}{ll}
A & B \\
C & D
\end{array}\right] \\
P(S) & =\left[\begin{array}{ll}
A_{s} & B_{s} \\
C_{s} & D_{s}
\end{array}\right] .
\end{aligned}\right.
$$

Now, the weighting matrix can be written as;

$$
W_{n}(S)=\left[\begin{array}{ll}
A_{W_{n}} & B_{W_{n}} \\
C_{W_{n}} & D_{W_{n}}
\end{array}\right] .
$$

Now, the organized controller form is expressed as;

$$
\left\{\begin{aligned}
A= & {\left[\begin{array}{ccc}
A_{W_{1}} & 0 & B_{W_{1}} C_{s} \\
0 & A_{W_{2}} & B_{W_{2}} C_{s} \\
0 & 0 & A_{W_{3}}
\end{array}\right] * A_{s} } \\
B= & {\left[\begin{array}{cc}
B_{W_{1}} & B_{W_{1}} D_{s} \\
0 & B_{W_{2}} D_{s} \\
0 & B_{W_{3}} D_{s}
\end{array}\right] * B_{s} } \\
C= & {\left[\begin{array}{ccc}
C_{W_{1}} & 0 & D_{W_{1}} C_{s} \\
0 & C_{W_{2}} & D_{W_{2}} C_{s} \\
0 & 0 & C_{W_{3}}
\end{array}\right] } \\
D= & {\left[\begin{array}{cc}
D_{W_{1}} & D_{W_{1}} D_{s} \\
0 & D_{W_{2}} D_{s} \\
1 & D_{s}
\end{array}\right] }
\end{aligned}\right.
$$

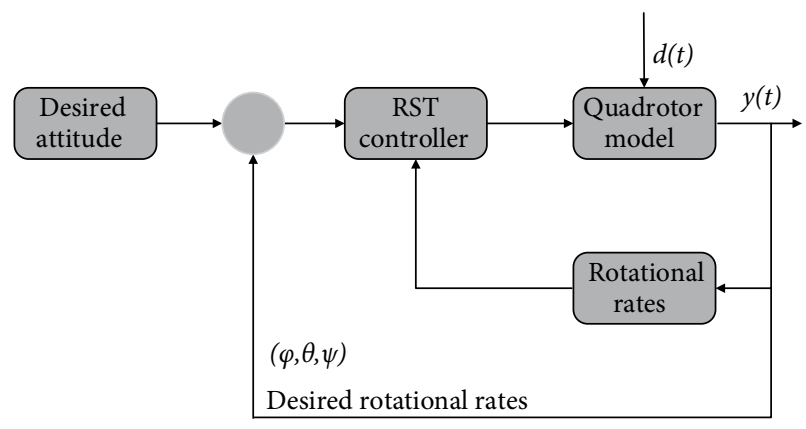

FIGURE 3: RST Controller based attitude control block diagram.

\section{Modeling of Control Objects}

The direction/position of the UAV can be achieved by changing the velocity or speed of its rotors. Now, in order to realize the translational and rotational motion of quad-rotor UAV, it has 6 DOF and less number of actuators that's why it falls in the category of under actuated system. To design the controller, the attitude angles or Euler angles of the model are simplified to a single input and single output (SISO) model. By neglecting the coupling between DOF's of quad rotor UAV the roll angle model of quad rotor can be obtained from the transfer function of actuator commanded to the roll angle.

Remark III: According to the Newton Euler's formulation, the nonlinear equation of motion is linearized by equilibrium points. The relationship between the torque and Euler angles is obtained as;

$$
\tau=I_{r} \varphi,
$$

where " $\tau$ " torque vector, " $I_{r}$ " is moment of inertia and " $\varphi$ " is the roll angle. Now the Laplace transformation of the above equation is;

$$
\tau(s)=I_{r}(s) \cdot \varphi(s) \cdot s^{2} .
$$

The relationship between the velocity of actuator, difference control and torque can be approximated as a step inertia link;

$$
\tau(s)=\frac{k}{\left(1+\mu_{1} s\right)} u_{n}(s),
$$

where " $k$ " is the proportional coefficient, " $\mu$ " is a time constant its velocity is calculated by $u_{n}(s)$;

$$
G_{\varphi_{n}}=\frac{\varphi(s)}{u_{n}(s)}=\frac{k}{I_{r}\left(1+\mu_{1} S\right) S^{2}} .
$$


In Equation (22), the roll angle model can be expressed as;

$$
\left\{\begin{array}{l}
{\left[\begin{array}{l}
\dot{x}_{1} \\
\dot{x}_{2} \\
\dot{x}_{3}
\end{array}\right]=\left[\begin{array}{ccc}
\frac{1}{\mu_{1}} & 0 & 0 \\
1 & 0 & 0 \\
0 & 1 & 0
\end{array}\right]\left[\begin{array}{l}
x_{1} \\
x_{2} \\
x_{3}
\end{array}\right]+\left[\begin{array}{c}
\frac{k}{I_{r} \mu_{1}} \\
0 \\
0
\end{array}\right] u} \\
y=\left[\begin{array}{lll}
0 & 0 & 1
\end{array}\right]\left[\begin{array}{l}
x_{1} \\
x_{2} \\
x_{3}
\end{array}\right]
\end{array}\right.
$$

The unknown parameters of the model are identified by least square method combined with actual flight data. The results of identified parameters are as follows.

$$
\left(\begin{array}{c}
\frac{-1}{\mu_{1}} \\
\frac{k}{I_{r} \mu_{1}}
\end{array}\right)=\left(\begin{array}{c}
-14.68 \\
4.6971
\end{array}\right) .
$$

\section{Selection of Attitude $H_{\infty}$ Controller Design Based on Mixed Sensitivity}

5.1. The Weighting Function. In mixed sensitivity design, the weighting of sensitivity determines the magnitude and gain of its sensitivity function. Now the weighting function " $W$ " is the norm boundary of the instruction error ratio and the disturbance or rejection ratio.

Remark IV: By selecting the weighting function the amplitude and cutoff frequency of robust controller, the following requirements should be in considerations.

(1) To track the desired response precisely and accurately the gain of weighting function " $W_{1}$ " should be large in low frequency domain. On the other hand, there are no any requirements for the high frequency band.

(2) Due to the high frequency constraints in the system, it may causes uncertainty in the system model. The weighting function " $W_{2}$ " should select a function with large gain and high frequency without any error and attenuation.

(3) The arrangements of the weighting function in the system is capable of proper tuning the order of the controller. Now " $W_{3}$ " can control the order of the controller and in favor of tracking the system at desired response respectively.

$$
\left\{\begin{array}{l}
W_{1}(S)=\frac{S+300}{6 S+90} \\
W_{2}(S)=\frac{S+5}{0.01 S+100} \\
W_{3}(S)=\frac{S+10}{0.01 S+500}
\end{array}\right.
$$

5.2. Selection the Gains of Controller. The attitude angle model of a quad-rotor aircraft is already written in Equation (11). The angle model for the controlling the position of quad-rotor USV can be written as;

$$
\left\{\begin{array}{l}
\dot{x}(t)=A_{P} x(t)+B_{P} u \\
Y(t)=C_{P} x(t)+D_{P} u
\end{array}\right.
$$

TABLE 1: Constants of quad-rotor UAV.

\begin{tabular}{lcc}
\hline Constants & Value & Unit \\
\hline $\mathrm{m}$ & 2.5 & $\mathrm{~kg}$ \\
$I_{x}$ & 0.3998 & $\mathrm{~m}^{\wedge} 2 \cdot \mathrm{kg}$ \\
$I_{y}$ & 0.479 & $\mathrm{~m}^{\wedge} 2 \cdot \mathrm{kg}$ \\
$I_{z}$ & 0.887 & $\mathrm{~m}^{\wedge} 2 \cdot \mathrm{kg}$ \\
$l$ & 0.25 & $\mathrm{~m}$ \\
\hline
\end{tabular}

Now the generalized object "G" for the mixed/hybrid control sensitivity problem of RST controller which was derived in Section 2. It is combined with Equations (25) and (26) to obtain a model of quad-rotor UAV. The generalized object of attitude angle is solved by $H_{\infty}$ controller that is based on Riccati equation. The controller is obtained by solving the performance index " $\beta$ " is taken as $0.9922 H_{\infty}$.

The obtained controller is

$$
K=\left[\begin{array}{ll}
K_{11} & K_{12} \\
K_{21} & K_{22}
\end{array}\right]
$$

Now,

$$
\begin{aligned}
& k_{11}=\left[\begin{array}{ccccc}
-33.8 & -18.8 & 28.2 & -0.003 & 5.03 \\
18.75 & 0 & -63.03 & 0 & 0 \\
0 & 0.25 & 6.7 & 0 & 0 \\
0 & 0 & 0 & 3.33 & 0 \\
-3.15 & -5.75 & 172.8 & -19.2 & 5.93
\end{array}\right] \\
& k_{12}=\left[\begin{array}{c}
514.83 \\
-1149.4 \\
-81.73 \\
-42.6 \\
0
\end{array}\right] \\
& k_{21}=\left[\begin{array}{c}
-0.001 \\
-0.0002 \\
-0.0082 \\
0.0036 \\
1.0539
\end{array}\right] ; \\
& k_{22}=0 .
\end{aligned}
$$

\section{Simulation Results and Discussions}

This section defines the validity and efficiency of our designed robust hybrid controller. Table 1, discussed the constraints of quad-rotor UAV. The complete model of UAV is divided into two sub models, one is the outer loop for altitude control and the other one is the inner loop for attitude control. The combination of both loops could make the successful flight as per the desired direction and position. The initial errors in the model are controlled and stabilized by rotational sub system. However, the attitude angles are converging to zero to make sure that the flight is at hovering mode. The transient and steady state switching of inputs are laid between 0 and $3 \mathrm{sec}$ along with certain oscillations. The desired altitude for hovering scenario is said to unity, which is shown in Figure 4(c). The other positional axis that are equal to 0 . Figures $5-7$, shows the translational, rotational and attitude angle responses of the simulated model. 


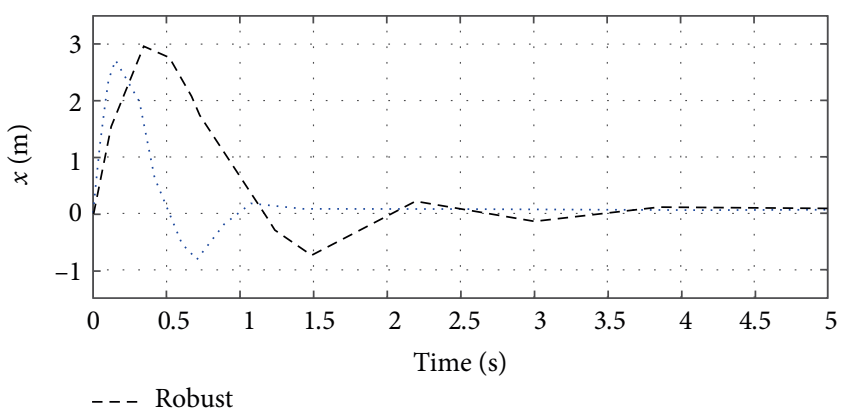

Hybrid

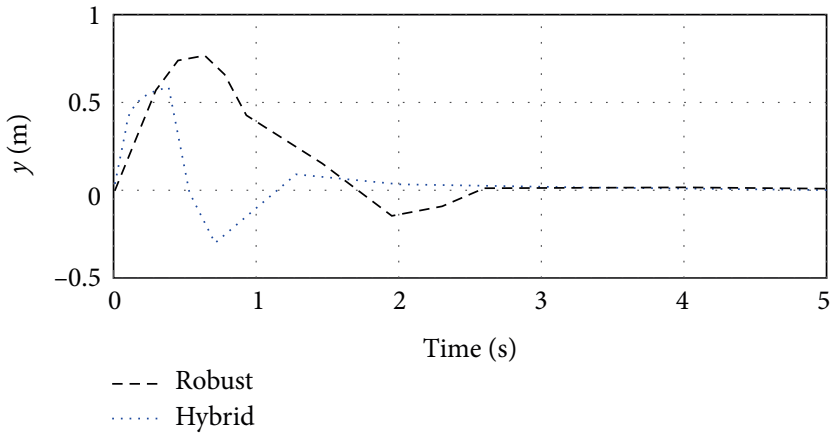

(b)

(a)

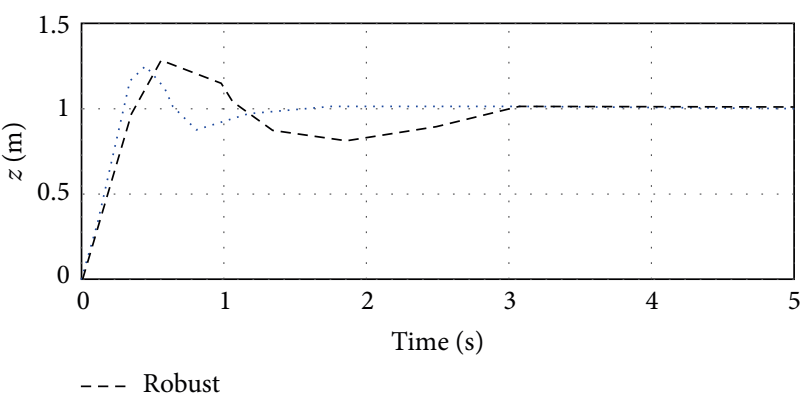

Hybrid

(c)

Figure 4: (a) The $x$-position of UAV. (b) The $y$-position of UAV. (c) The $z$-position of UAV.

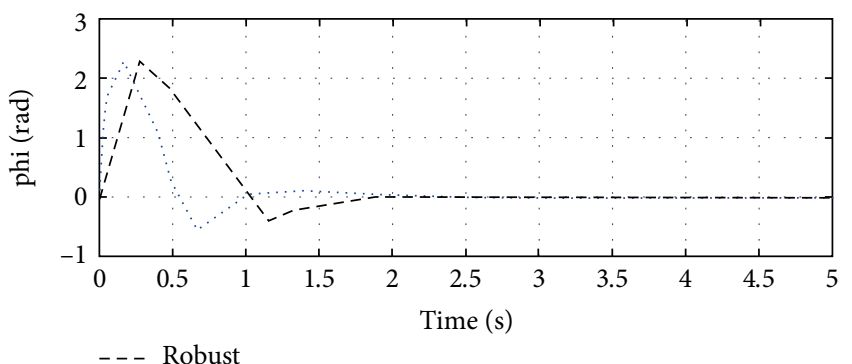

Hybrid

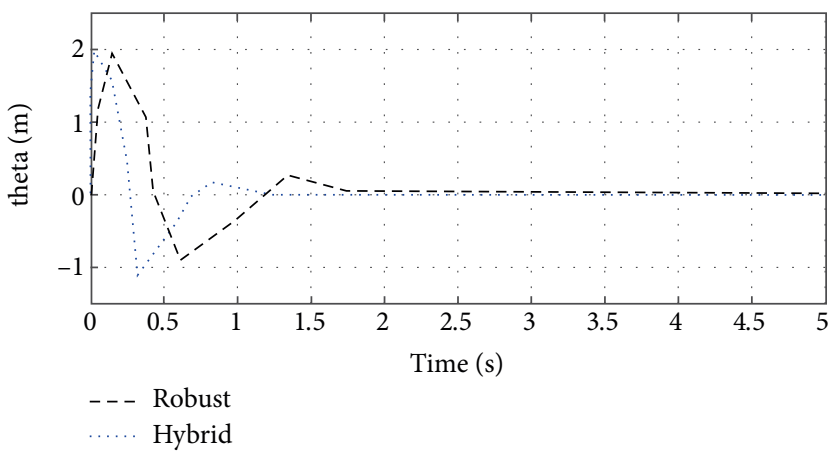

(b)

(a)

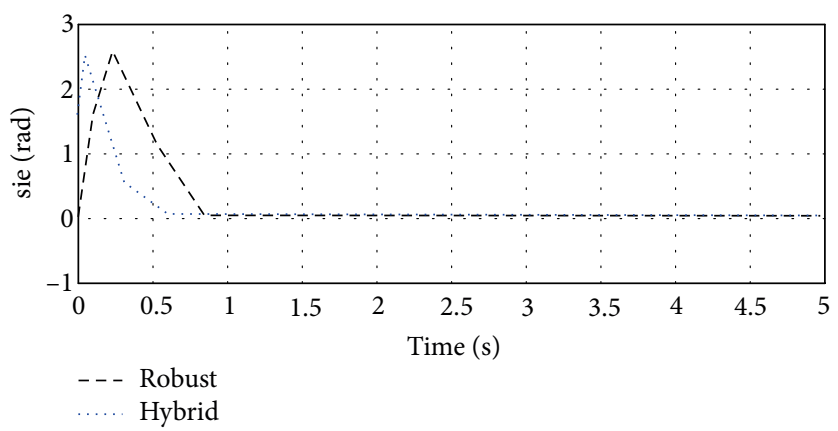

(c)

Figure 5: (a) The Euler angles phi of UAV. (b) The Euler angles theta of UAV. (c) The Euler angle sie of UAV. 


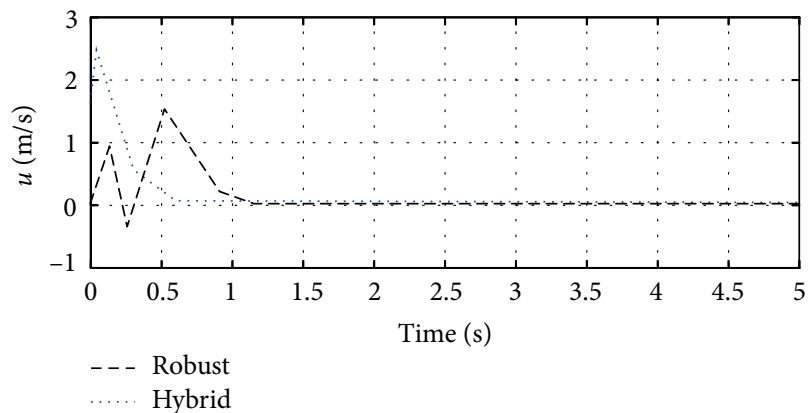

(a)

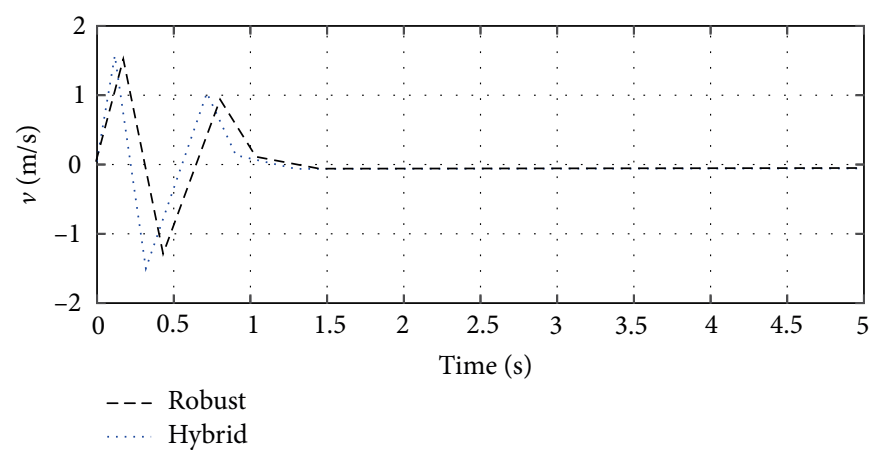

(b)

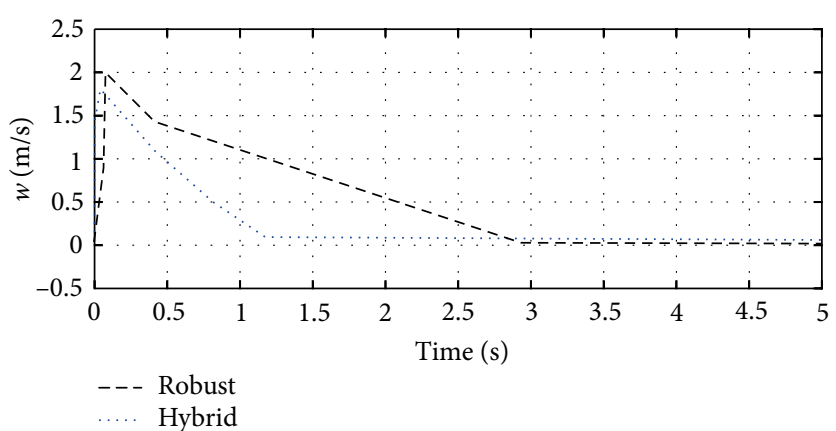

(c)

FIgURE 6: (a) The translational velocity $u(\mathrm{~m} / \mathrm{s})$. (b) The translational velocity $v(\mathrm{~m} / \mathrm{s})$. (c) The translational velocity $w(\mathrm{~m} / \mathrm{s})$.

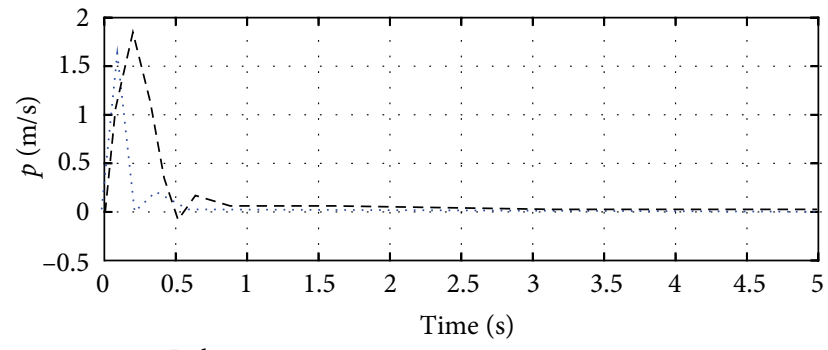

Hybrid

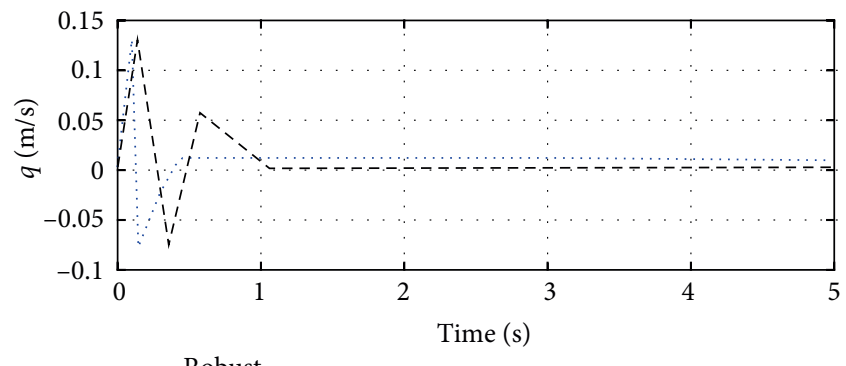

--- Robust

Hybrid

(a)

(b)

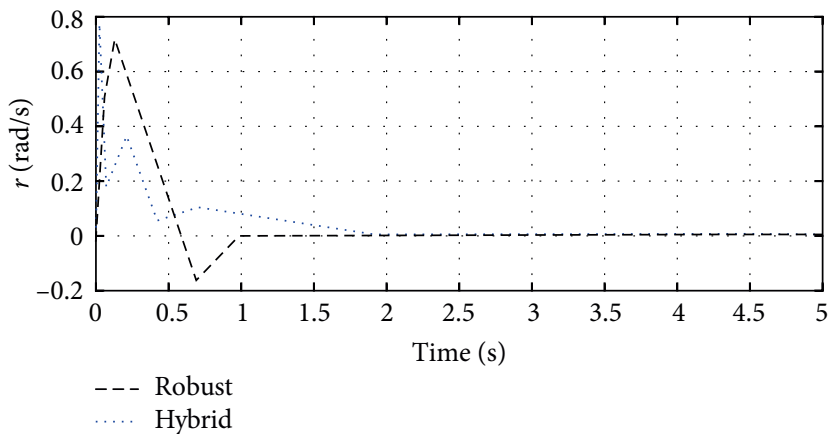

(c)

Figure 7: (a) The rotational velocity $p(\mathrm{~m} / \mathrm{s})$. (b) The rotational velocity $q(\mathrm{~m} / \mathrm{s})$. (c) The rotational velocity $r(\mathrm{~m} / \mathrm{s})$.

The sampling period of overall scenario was set to $0.25 \mathrm{sec}$ for every simulation which was done in ten sec. The designed robust hybrid method converges to the referred path robustly as compared to robust controller which is shown in Figures
4-7. The initial position of the UAV is $(0,0,0) \mathrm{m}$ and its hovering position is $(0,0,1) \mathrm{m}$.

The initial conditions of attitude angles have several oscillations in the start, after some seconds it all converges to the 
zero, as per the desired input command. For hovering scenario by applying the trim point condition the height or altitude of UAV is fixed at $1 \mathrm{~m}$ height that's why the above velocity components goes to zero due to no moment in the dynamics of flight which is seen in Figures 6 and 7 respectively.

The simulated results from Figures 4-7 showed that the designed controller has better transient and steady state responses as compared to robust controller.

\section{Conclusion}

In this paper a new and novel hybrid control scheme is designed to control the altitude and attitude of UAV. The designed control algorithm is able to control the nonlinear behavior of UAV. Furthermore, the inner loop (attitude control) and the outer loop altitude control loops are used to achieve the desired height and maintain hovering state by using trim point condition. The validity of the controller is proven by using computer-based simulation, which illustrates the efficiency of the hybrid algorithm over robust controller. It shows that it has best steady state response with minimum overshoot to achieve the hovering state. In the end, the selected model of quad-rotor UAV converges quickly conferring to the desired path effectively.

\section{Data Availability}

The data of robust controller for quadrotor is provided by Engr. Zain Anwar Ali and Prof. Daobo Wang, S\&MC laboratory [23], Nanjing University of Aeronautics and Astronautics.

\section{Conflicts of Interest}

The authors declare that they have no conflicts of interest.

\section{References}

[1] Adam C. Watts, Vincent G. Ambrosia, and Everett A. Hinkley, "Unmanned aircraft systems in remote sensing and scientific research: classification and considerations of use," Remote Sensing, vol. 4, no. 6, pp. 1671-1692, 2012.

[2] S. Karma, E. Zorba, G. C. Pallis et al., "Use of unmanned vehicles in search and rescue operations in forest fires: advantages and limitations observed in a field trial," International Journal of Disaster Risk Reduction, vol. 13, pp. 307-312, 2015.

[3] Ismael Colomina and Pere Molina, "Unmanned aerial systems for photogrammetry and remote sensing: a review," ISPRS Journal of Photogrammetry and Remote Sensing, vol. 92, pp. 79-97, 2014.

[4] B. M. Waterman, B. H. Pasculano, J. A. Goldsberry et al., Vertical Take-Off and Landing Autonomous Aircraft Design, Worcester Polytechnic Institute, Worcester, MA, USA, 2019.

[5] L. A. Young, A Multi-Modality Mobility Concept for a Small Package Delivery UAV, NASA Ames Research Center, Moffett Field, CA, USA, 2017.

[6] L. Matikainen, M. Lehtomäki, E. Ahokas et al., "Remote sensing methods for power line corridor surveys," ISPRS Journal of Photogrammetry and Remote Sensing, vol. 119, pp. 10-31, 2016.
[7] M.Hoffman, "Multi-sensor track classification in rotorcraft pilot's associate data fusion," in Annual Forum Proceedings-American Helicopter Society, pp. 271-280, American Helicopter Society, Fairfax, VI, USA, 1997.

[8] P. Liu, A. Y. Chen, Y.-N. Huang et al., "A review of rotorcraft unmanned aerial vehicle (UAV) developments and applications in civil engineering," Smart Structures and Systems, vol. 13, no. 6, pp. 1065-1094, 2014.

[9] A. Kube, "Rotorcraft winch lighting device and method of operating a rotorcraft winch lighting device," U.S. Patent 9,994,333, issued June 12, 2018.

[10] B. S. Kim and A. J. Calise, "Nonlinear flight control using neural networks," Journal of Guidance, Control, and Dynamics, vol. 20, no. 1, pp. 26-33, 1997.

[11] W. S. Levine, The Control Systems Handbook: Control System Advanced Methods, CRC Press, Boca Raton, FL, USA, 2018.

[12] Z. T. Dydek, A. M. Annaswamy, and E. Lavretsky, "Adaptive configuration control of multiple UAVs," Control Engineering Practice, vol. 21, no. 8, pp. 1043-1052, 2013.

[13] B. Erginer and A. Erdinc, "Modeling and PD control of a quadrotor VTOL vehicle," in 2007 IEEE Intelligent Vehicles Symposium, pp. 894-899, IEEE, Las Vegas, NV, USA, 2007.

[14] C. Zhang, X. Zhou, H. Zhao, A. Dai, and Z. Huiling, “Threedimensional fuzzy control of mini quadrotor UAV trajectory tracking under impact of wind disturbance," in 2016 International Conference on Advanced Mechatronic Systems (ICAMechS), pp. 372-377, IEEE, Melbourne, Australia, 2016.

[15] I. D. Landau, "The RST digital controller design and applications," Control Engineering Practice, vol. 6, no. 2, pp. 155-165, 1998.

[16] L. Fortuna and M. Frasca, Optimal and Robust Control: Advanced Topics with MATLAB', CRC Press, Boca Raton, FL, USA, 2012.

[17] Z. A. Ali, D. Bo Wang, and M. Aamir, "Design a robust RST controller for stabilization of a tri-copter UAV," Pakistan Journal of Engineering, Technology \& Science, vol. 5, no. 1, 2016.

[18] Z. Ali, D. Wang, and M. Aamir, "Fuzzy-based hybrid control algorithm for the stabilization of a tri-rotor UAV," Sensors, vol. 16, no. 5, p. 652, 2016.

[19] Z. A. Ali, D. Wang, S. Masroor, and M. S. Loya, "Attitude and altitude control of trirotor UAV by using adaptive hybrid controller," Journal of Control Science and Engineering, vol. 2016, pp. 1-12, 2016.

[20] Z. A. Ali, D. B. Wang, M. Aamir, and S. Masroor, "MRAC base robust RST control scheme for the application of UAV," International Journal of Modelling, Identification and Control, vol. 28, no. 3, pp. 232-244, 2017.

[21] Z. A. Ali, D.-B. Wang, R. Javed, and A. Akbar, "Modeling \& controlling the dynamics of tri-rotor UAV using robust RST controller with MRAC adaptive algorithm," International Journal of Control and Automation, vol. 9, no. 3, pp. 61-76, 2016.

[22] Ashfaq Ahmad Mian and Wang Daobo, "Modeling and backstepping-based nonlinear control strategy for a 6 DOF quadrotor helicopter," Chinese Journal of Aeronautics, vol. 21, no. 3, pp. 261-268, 2008.

[23] A. Alaimo, V. Artale, C. Milazzo, A. Ricciardello, and L. Trefiletti, "Mathematical modeling and control of a hexacopter," in 2013 International Conference on Unmanned Aircraft Systems (ICUAS), pp. 1043-1050, IEEE, USA, Atlanta, 2013. 